Background Pooling resources for the development and dissemination of guidelines receives important consideration due to the extensive amount of expertise, money and staff time needed within an organisation. Partnered guidelines may increase the administrative cost and timeline of development, but is positively offset in the value partnering brings in the ultimate success and implementation.

Context Over the last three years we formally partnered with other professional medical societies in over $70 \%$ of our guidelines, learning many core and nuanced components of a successful jointly-developed guideline. We share those lessons learned with GIN members.

Description of Best Practice We tackle critically important aspects of joint collaborations, beginning with the determination of appropriate partners. We explore the creation of a solid memorandum of understanding (MOU), addressing questions like: How will we select panel membership and manage their conflicts of interest? What grading system will we utilise with an evidence-based guideline or consensus conference? How will we approach our respective organisation's approval process? What is needed to produce a simultaneous joint publication between journals? How will we disseminate effectively to our target audiences? What is our future plan for a revision? And the ultimate questions - How do we cost share and work share in the development equally?

Lessons for Guideline Developers, Adaptors, Implementers, and/or Users These experiences will help guideline developers create a framework for partnered collaborations, balancing value gained in partnership versus challenges realised in completion and resourcing.

\section{STRATEGIES FOR CLINICAL EXPERT AND STAKEHOLDER INVOLVEMENT IN UPDATING AND IMPLEMENTING CLINICAL GUIDANCE FOR NEW AND EMERGING MEDICAL INTERVENTIONS}

E Loughren. Kaiser Permanente, Southern California Permanente Medical Group, USA

\section{0:1136/bmjgs-2013-002293.76}

Background Lack of sufficient clinical expert and stakeholder involvement in the routine updating of guidance on new and emerging medical interventions can lead to inefficient use of resources and inadvertently create unnecessary barriers to implementation.

Context An evidence services unit within a large health care organisation developed a stakeholder-centred process for rapid updating and implementation of guidance related to the use of breast magnetic resonance imaging (MRI).

Description of Best Practice The process focused on the following: 1) asking clinical stakeholders to identify the indications for which use of breast MRI remained unresolved or controversial; 2) conducting a search for high-quality systematic reviews and clinical trials for the specific indications, and contacting wellknown external content experts to identify unpublished evidence; 3) obtaining data on the organisation's current breast MRI utilisation and practice variation; 4) engaging experts/stakeholders in guidance development and revision based on current utilisation/practice variation compared to findings from the evidence review; 5) obtaining endorsement of guidance and commitment to implementation efforts from clinical opinion leaders and other stakeholders; and 6) initiating routine monitoring and feedback on breast MRI use.
Lessons for Guideline Developers, Adaptors, Implementers, and/or Users Limiting evidence updates to controversial or unresolved areas of clinical practice, engaging stakeholders in guidance development based on a review of current utilisation data and scientific evidence, and engaging key opinion leaders and stakeholders in implementation and performance monitoring leads to more efficient use of resources, stronger implementation and improved performance.

\section{GUIDELINE DEVELOPMENT TOOL (GDT) - WEB-BASED SOLUTION FOR GUIDELINE DEVELOPERS AND AUTHORS OF SYSTEMATIC REVIEWS}

1) Brozek, ${ }^{2} E$ Akl, ${ }^{3} Y$ Falck-Ytter, ${ }^{4} \mathrm{P}$ Kunstman, ${ }^{5}$ J Meerpohl, ${ }^{1} \mathrm{R}$ Mustafa, ${ }^{4} \mathrm{~A}$ Nowak, ${ }^{6} \mathrm{~A}$ Oxman, ${ }^{1} \mathrm{~N}$ Santesso, ${ }^{1} \mathrm{~W}$ Wiercioch, ${ }^{1} \mathrm{H}$ Schünemann. ${ }^{1}$ McMaster University, Hamilton, Canada; ${ }^{2}$ American University of Beirut, Beirut, Lebanon; ${ }^{3}$ Case Western Reserve University, Cleveland, USA; ${ }^{4}$ Synaway, Krakow, Poland; ${ }^{5}$ Universitätsklinikum Freiburg, Freiburg, Germany; ${ }^{6}$ Norwegian Knowledge Centre for the Health Services, Oslo, Norway

\section{0:1136/bmjgs-2013-002293.77}

Background Guideline developers and other health care decision makers benefit from following a structured process of specifying the health care questions they intend to answer and the outcomes of interest, assessing the confidence in the available evidence, gathering information about the values and preferences of the target population, and presentation of their results and decisions to the target users. Many guideline developers use the GRADE Profiler (GRADEpro) software used to conduct this work.

Context GRADE's approach is currently being further defined in the DECIDE (Developing and Evaluating Communication Strategies to Support Informed Decisions and Practice Based on Evidence) project.

Description of Best Practice The Guideline Development Tool (GDT) is the extension of the GRADE Profiler (GRADEpro) software. The GDT provides an integrated platform-independent web-based solution for health care decision makers offering support for the whole process of making decisions and developing recommendations including question formulation, generation and prioritisation of outcomes, support for teamwork, management of potential conflicts of interest, presentation of results (including the functionality of GRADEpro) and decision support. We tested the software with individual users and in workshops as well as in guideline development processes.

Lessons for Guideline Developers, Adaptors, Implementers, and/ or Users Following a structured and systematic process, transparency and clarity of presentation facilitates the use of results of systematic reviews and facilitates development, updating and adaptation of evidence-based recommendations and decisions. Storing all information in a uniform, structured, transparent and annotated way also greatly facilitates updating and adaptation of systematic reviews and guidelines.

\section{IDENTIFYING FACTORS PREDICTIVE OF MANAGING PATIENTS WITH LOW BACK PAIN WITHOUT USING X-RAYS AMONG NORTH AMERICAN CHIROPRACTORS: APPLYING PSYCHOLOGICAL THEORIES TO EVIDENCE-BASED CLINICAL PRACTICE}

\footnotetext{
${ }^{1,2} \mathrm{~A}$ Bussières, ${ }^{3} \mathrm{~J}$ Francis, ${ }^{3} \mathrm{~A}$ Patey, ${ }^{4} \mathrm{M}$ Gagnon, ${ }^{5} \mathrm{~A}$ Sales, ${ }^{6} \mathrm{M}$ Eccles, ${ }^{7} \mathrm{~L}$ Lemyre, ${ }^{4} \mathrm{G}$ Godin, 8J Grimshaw. 'School Physical Occupational Therapy, Faculty of Medicine/McGill University, Montreal, Canada; ${ }^{2}$ Département Chiropratique, Université du Québec à
} 
Trois-Rivières, Trois-Rivières, Canada; ${ }^{3}$ Health Services Research and Management, City University London, London, UK; ${ }^{4}$ Faculté des sciences infirmières, Université Laval, Québec, Canada; ${ }^{5}$ School of Nursing, University of Michigan, Michigan, USA; ${ }^{6}$ Centre for Health Services Research, University of Newcastle upon Tyne, Newcastle, UK; ${ }^{7}$ School of Psychology, Faculty of Social Sciences, University of Ottawa, Ottawa, Canada; ${ }^{8}$ Department of Medicine, Ottawa Hospital Research Institute, University of Ottawa, Ottawa, Canada

\section{0:1136/bmjgs-2013-002293.78}

Background This study aimed to identify theoretically based modifiable factors that predict whether chiropractors manage patients with low back pain without ordering lumbar x-rays.

Methods A mailed survey with psychological measures was collected from a random sample of Ontario (Canada) and Practice Network (US) chiropractors. The outcome measures were behavioural intention and behavioural simulation (scenario decisionmaking). Explanatory variables included constructs from motivational theories (Theory of Planned Behaviour (TPB), Theory of Interpersonal Behaviour (TIB)), action theories (Operant Learning Theory (OLT) and Planning (action and coping)), and two other constructs: personal moral norm and habit as measured by the Self-Reported Habit Index (SRHI). Multiple regression analyses examined the predictive value of each theoretical model individually for simulation and intention outcomes.

Results 31\% of North American chiropractors returned completed questionnaires. Overall, TPB and TIB, followed by personal moral norms and OLT best explained behavioural simulation. Theory level variance explained among Ontario and US chiropractors was respectively: TPB 59\%; 52.0\%, TIB $57 \%$; $54.0 \%$, personal moral norm 49\%; 46.0\%, OLT 49\%; $52.0 \%$, action planning 28\%; 29\%, and SRHI 42\%; 48\%. Constructs from TPB and TIB best explained behavioural intention. Theory level variance explained was respectively: TPB $85 \%$; $74 \%$, TIB; 83\%; 81\%, OLT 62\%; 69\%, and SRHI 59\% and $52 \%$ for SRHI.

Conclusion These models explained up to $59 \%$ of the variation in behavioural simulation and up to $85 \%$ in intention to manage back pain patients without $\mathrm{x}$-rays. Results may inform development of theory-based behaviour change interventions to implement imaging guideline recommendations among North American chiropractors. These models explained up to $59 \%$ of the variation in behavioural simulation and up to $85 \%$ in intention to manage back pain patients without x-rays. Results may inform development of theory-based behaviour change interventions to implement imaging guideline recommendations among North American chiropractors.

\section{DEVELOPMENT OF FIRST AID GUIDELINES FOR RECREATIONAL ATHLETES BY BELGIAN RED CROSS- FLANDERS}

${ }^{1} \mathrm{~N}$ Pauwels, ${ }^{1} \mathrm{E}$ De Buck, ${ }^{2,3} \mathrm{P}$ Viroux, ${ }^{4} \mathrm{~J}$ Bellemans, ${ }^{5} \mathrm{R}$ Meeusen, ${ }^{6} \mathrm{~K}$ Peers, ${ }^{7} \mathrm{~K}$ Verhelst, ${ }^{8} \mathrm{~T}$ Dieltjens. 'Centre for Evidence-Based Practice (CEBaP), Belgian Red Cross-Flanders, Mechelen, Belgium; ${ }^{2}$ Athlete's Care BVBA, Antwerp, Belgium; ${ }^{3}$ Bloso, Brussels, Belgium; ${ }^{4}$ University Hospital campus Pellenberg, Catholic University Leuven, Leuven, Belgium; ${ }^{5}$ Vrije Universiteit Brussel, Brussels, Belgium; ${ }^{6}$ University Hospital campus Gasthuisberg, Catholic University Leuven, Leuven, Belgium; ' 7 Humanitarian Services, Belgian Red crossFlanders, Mechelen, Belgium

\section{0:1136/bmjqs-2013-002293.79}

Background Belgian Red Cross-Flanders (BRCFl) is active in many fields including first aid training, for which BRCFl develops evidence-based guidelines according to AGREE II.
Objectives Development of evidence-based recommendations for recreational athletes who perform one of 15 sport disciplines, defined by popularity, medical costs and injury risk in Flanders (Belgium). Initiatives concerning wording and accessibility were taken to develop implementable guidelines adapted for laypeople. These guidelines will also be used by the Flemish Government to update its information sources.

Methods 10 databases for guidelines, systematic reviews or individual studies were searched and draft recommendations were formulated based on the best current evidence. The quality of evidence was assessed using the GRADE approach. A guideline development group, including a multidisciplinary expert panel (co-authors PV, JB, RM and KP), discussed the draft recommendations while taking into account the evidence, and validated the final recommendations.

Results 32 systematic reviews and 2 guidelines that met the methodological criteria were identified as valuable source of studies. Additionally, 73 individual studies were included. The overall quality of the body of evidence varied from moderate to very low. Recommendations, written in active and explicit wording, were organised in a structure in order to be searchable via sports discipline, anatomical localisation, type of intervention, and injury.

Discussion Preferences of the target group were taken into account when selecting the sport disciplines and when formulation the evidence-based recommendations.

Implications for Guideline Developers/Users Involving the target population is an added value for developing an implementable evidence-based guideline.

\section{EVIDENCE BASED TREATMENT GUIDELINES: AT WORK IN A MICROCOSM}

1,3,4,6,9,10p Whelan, 1,2,5,6,7,9,10p Denniston. 'Work Loss Data Institute, LLC, Encinitas, USA; ${ }^{2}$ American Academy of Disability Evaluating Physicians (AADEP), Chicago, USA; ${ }^{3}$ Insurance Council of Texas (ICT), Austin, USA; ${ }^{4}$ Industry Education Council (IEC), Albany, USA; ${ }^{5}$ American College of Occupational \& Environmental Medicine (ACOEM), Elk Grove, USA; ${ }^{6}$ New York Self-Insurers Association (NYSIA) Buffalo, NY, USA; ${ }^{7}$ American Association of State Compensation Insurance Funds (AASCIF) Towson, USA; 8 International Association of Industrial Accident Boards (IAIABC) Madison, WI, USA; ${ }^{9}$ Business Council of New York Albany, NY USA; ${ }^{10}$ New York Claims Association New York, NY, USA

\section{0:1136/bmjqs-2013-002293.80}

Background Medical treatment under workers' compensation represents just a miniscule portion (about 3 percent) of total medical costs in the US Furthermore, legislation and rules are determined autonomously by each state. Worker' comp medical care, and the outcomes of that care, in each state theoretically can represent a microcosm of what could be achieved in an entire country. Impact of Evidence-based Treatment Guidelines: A trend began in 2003, starting with California, for states to consider adopting Evidence-Based Treatment Guidelines as a mechanism to insure timely and quality care for injured workers by following the least invasive, most-effective treatments today's science has to offer. Bi-products to the effective implementation of EBTG, include earlier return to work, better outcomes resulting in reduced indemnity costs, less friction in the system (providers know what treatments are authorised and will be paid for), fewer episodes of over-utilisation of services, and decreased medical costs, benefiting employers, insurers, providers and business. Outcomes where true EBTG have been implemented: Ohio adopted EBTG in 2003. A Pilot conducted in 2005 showed a 